\title{
The relationship between tumor volume changes and serial plasma osteopontin detection during radical radiotherapy of non-small-cell lung cancer
}

\author{
CHRISTIAN OSTHEIMER*, FRANZISKA SCHWEYER*, THOMAS REESE, \\ MATTHIAS BACHE and DIRK VORDERMARK
}

Department of Radiation Oncology, Martin Luther University Halle-Wittenberg, D-06120 Halle (Saale), Germany

Received May 18, 2016; Accepted July 28, 2016

DOI: $10.3892 / 01.2016 .5104$

\begin{abstract}
The prognostic quality of increased osteopontin (OPN) plasma levels has been demonstrated for the chemotherapy and surgery of lung cancer. There is also evidence in the literature that tumor volume impacts prognosis in definitive radiotherapy (RT) of (lung) cancer. We previously demonstrated that elevated plasma levels of OPN before, and increasing OPN plasma levels after RT significantly correlate with survival and outcome after curative-intent RT of non-small-cell lung cancer (NSCLC). Tumor volume was also associated with prognosis. The present prospective clinical study investigated the prognostic interrelation of OPN plasma levels and tumor volume and their changes in the radical RT of NSCLC. We evaluated a subset of patients $(n=27)$ with inoperable, non-metastasized NSCLC of the previously published patient collective. Patients were treated with radical radiochemotherapy (2 Gy ad 66 Gy). OPN plasma concentrations were determined by ELISA before (t0), at the end (t1), and 4 weeks after RT (t2). GTV was delineated PET- and CT-correlated before RT (GTV1) and after 40 Gy (GTV2). The course of OPN during and after RT and the change of GTV during RT was monitored over time and correlated with prognosis. Median GTV2 after 40 Gy $(63 \mathrm{ml})$ was significantly lower than pre-RT GTV1 (90 ml, P<0.0001). Median OPN before ( $\mathrm{t} 0$ ), at the end of ( $\mathrm{t} 1$ ) and four weeks after RT (t2) was 846, 777 and $624 \mathrm{ng} / \mathrm{ml}$ and not significantly different. GTV significantly declined by $39 \mathrm{ml}$ during $\mathrm{RT}(\mathrm{P}<0.0001)$ and OPN non-significantly decreased by $56 \mathrm{ng} / \mathrm{ml}$ during (t0 to $\mathrm{t} 1$ ) and by $54 \mathrm{ng} / \mathrm{ml}$ after RT (t1 to t2). No correlations were determined between absolute OPN and GTV values or their relative
\end{abstract}

Correspondence to: Dr Christian Ostheimer, Department of Radiation Oncology, Martin Luther University Halle-Wittenberg, Ernst-Grube-Strasse 40, D-06120 Halle (Saale), Germany

E-mail: christian.ostheimer@uk-halle.de

*Contributed equally

Key words: NSCLC, osteopontin, radiotherapy, tumor volume, prognostic factors changes during RT. In univariate analysis, only GTV2 significantly predicted overall survival $(\mathrm{OS}, \mathrm{P}=0.03)$. In multivariate analysis, both OPN t1 $(\mathrm{P}<0.001)$ and GTV2 $(\mathrm{P}=0.001)$ remained significant predictors of OS. Relative OPN plasma level changes after (t1 to t2) and GTV changes during RT (GTV 1 to GTV 2) significantly predicted OS $(\mathrm{P}=0.02)$. The combination of absolute GTV values before RT (GTV1) and GTV changes during RT (GTV1 to 2) were significantly associated with OS in both uni- and multivariate analysis $(\mathrm{P}=0.03)$. The combination of absolute OPN plasma levels and their changes with GTV and its changes did not reach statistical significance. The lack of a significant correlation between OPN and GTV together with the finding that OPN and GTV remained independent predictors of survival outcome but were not associated with OS in combination supports the hypothesis that tumor volume (GTV) and OPN plasma levels (both their changes and absolute values) are not interrelated in terms of prognosis but do possess each parameter separately, a prognostic quality in the radical RT of NSCLC which justifies further prospective studies to validate these results.

\section{Introduction}

Lung cancer is a devastating disease and prognosis is still poor especially in locally advanced stages where the standard treatment is definite concurrent chemoradiotherapy (CCRT) $(1,2)$. Tumor hypoxia is a critical factor of the tumor microenvironment which negatively impacts response to CCRT in many human cancers, including lung cancer (3-7).

Predictive and prognostic factors which specifically identify patients with hypoxic and radioresistant tumors before radiotherapy (RT) who are likely to benefit from anti-hypoxic treatment would be valuable tools in the curative-intent RT of advanced non-small cell lung cancer (NSCLC) (8-10).

We previously demonstrated that elevated baseline plasma levels of the hypoxia-related proteins osteopontin (OPN), vascular-endothelial-growth-factor (VEGF) and carbonic anhydrase IX (CAIX) additively correlate with prognosis (11) and that particularly post-treatment OPN plasma level changes predict survival after radical CCRT of NSCLC (12).

Interestingly, we also found a significant association of OPN plasma levels with gross tumor volume (i.e. higher basal 
OPN plasma levels in patients with larger GTV) in a series of 69 NSCLC M0-stage patients treated with definite CCRT. In the literature, there is evidence that GTV has a clinically relevant impact on overall survival (OS) after CCRT of NSCLC (13-16).

This prospective clinical study investigated the potential prognostic interrelation of tumor volume and its changes with OPN plasma levels during CCRT of NSCLC and aimed to determine whether OPN plasma levels merely are a surrogate of radiation-induced tumor volume changes accounting for the prognostic effect of OPN.

\section{Patients and methods}

Patients. In this prospective study, a subset of 27 patients of the previously published patient collective $(11,12)$ was evaluated. Only patients with complete OPN and tumor volume data on different time points were analyzed. Inclusion criteria were age $\geq 18$ years, histologically confirmed, inoperable and untreated NSCLC without distant metastases (M0-stage only), indication for definitive RT as determined by multidisciplinary tumor board. The study protocol was approved by the ethics committee of the Medical Faculty of the Martin Luther University Halle-Wittenberg. Staging was performed according to the TNM classification of malignant tumors (7th edition). Written informed consent was obtained from all patients prior to enrollment into the trial. The local Ethics Committee of the University approved the study and all procedures were in accordance with the Helsinki Declaration of 1975 (as revised 2008).

Radiochemotherapy and volumetric parameters. Three-dimensional conformal RT (3D-RT) was administered normofractionated ( 5 fractions/week) with curative intent (66 Gy total dose, 2 Gy single dose; Siemens Primus, Germany). Chemotherapy consisted of cisplatin $\left(20 \mathrm{mg} / \mathrm{m}^{2}\right.$ body surface on day 1-5) and vinorelbine $\left(25 \mathrm{mg} / \mathrm{m}^{2}\right.$ body surface on day 1) in treatment week one and five (2 courses) if general performance status and comorbidities allowed it (i.e. no renal and hepatic function impairment, normal blood cell count). RT was computed tomography (CT) based (Siemens Lightspeed RT, Germany) and all patients received a positron emission tomography (PET)-scan (Philips Accel, USA) before RT. CT and PET images were merged and GTV was defined as the primary tumor and involved nodes (pathologic confirmed, highly suspicious by CT and PET). GTV was delineated by an experienced radiation oncologist at planning CT before RT (GTV1) and all image data was registered in the Oncentra Masterplan external beam planning software (Nucletron, USA) used for RT plan calculation. After $40 \mathrm{~Gy}$, a new planning CT was performed and GTV was re-contoured (GTV2 i.e. before the initiation of boost RT) and adjusted for atelectasis, pneumonia and pleural effusion (Fig. 1).

OPN plasma samples and follow-up. Plasma samples were collected from patients at the time of routine blood sampling prior to (t0), at the end ( $\mathrm{t} 1)$, and at four weeks after radiotherapy (t2). They were centrifuged and stored at $-80^{\circ} \mathrm{C}$ until assayed. OPN plasma concentration was determined by enzyme-linked-immunosorbent-assay (ELISA, Human Osteopontin Assay, IBL Ltd., Japan). Clinico-pathological and demographic information was taken from the patients' charts and follow-up of patients was carried out regularly at the Department of Radiation Oncology, University Hospital Halle, Germany. Survival status was continuously monitored in cooperation with local citizen registration offices.

Statistical analysis. All statistical analyses were performed using the PASW statistical software (ver. 18, SPSS, Inc., Chicago, IL, USA) and statistical significance was accepted with two-sided $\mathrm{P}<0.05$, and a statistical trend was defined as $\mathrm{P}<0.1$. Only patients with a minimal follow-up time of two years were evaluated.

Nonparametric tests (Mann-Whitney U, Kruskal-Wallis $\mathrm{H})$ evaluated the relationship of pre-RT GTV and OPN (t0) plasma levels with clinicopathological characteristics and Wilcoxon's test compared pre-treatment with post-treatment OPN plasma levels and GTV. OPN plasma levels are reported with median $\mathrm{ng} / \mathrm{ml}$ (range) and median GTV as $\mathrm{ml}$ (range). GTV changes were defined as absolute $\mathrm{ml}$ and $\%$ (GTV2-GTV1/GTV1). Absolute OPN and GTV values detected at different time points were analyzed as categorical variables with the median used as the cut-off value (i.e. $\geq$ median vs. <median). Relative OPN and GTV changes were incorporated into analyses as categorical variables with the median change $(\%)$ used as a cut-off value (i.e. $\geq$ median vs. <median) and with increasing vs. decreasing OPN and GTV, respectively. Pearson's test was applied to determine correlation between OPN plasma levels and GTV detected at different time points. Survival curves were generated using Kaplan-Meier analysis set to the primary endpoint overall survival (OS, from start of RT until death or last seen in follow-up) with the log-rank test to test for differences. Uni- and multivariate analyses were performed to identify prognostic factors for OS using the Cox proportional hazard model to calculate relative risk, hazard ratio and its $95 \%$ confidence interval (CI) (17). Multivariate analyses incorporated variables shown to be significant or a statistical trend in univariate analysis in addition to known prognostic factors.

\section{Results}

Patient characteristics and their association with baseline OPN and GTV. Of the 27 patients, 20 patients (74\%) received CCRT while 7 patients $(26 \%)$ were treated with RT only. Median single dose was 2 Gy and total dose was 66 Gy (50-72 Gy). $41 \%$ of patients were diagnosed in UICC stage II and 59\% were in stage III. Table I provides patients' demographics, tumor and clinical characteristics. No associations between pre-RT GTV and clinical parameters were identified, however, pre-RT OPN (t0) was higher in patients with poorer lung function (i.e. lower FeV1) and in patients who reported significant weight loss. Pre-RT OPN was 914.6 (702.9-2441) $\mathrm{ng} / \mathrm{ml}$ in patients with low FeV1 compared to 626.5 (361-100.5) ng/ml in patients high FeV1 $(\mathrm{P}=0.002)$. Patients with weight loss had a median pre-RT OPN (t0) of 999.4 (702.9 to 1712.9$) \mathrm{ng} / \mathrm{ml}$ while it was 754.2 (357.4-2441) $\mathrm{ng} / \mathrm{ml}$ in those without significant weight loss $(\mathrm{P}=0.01)$. 

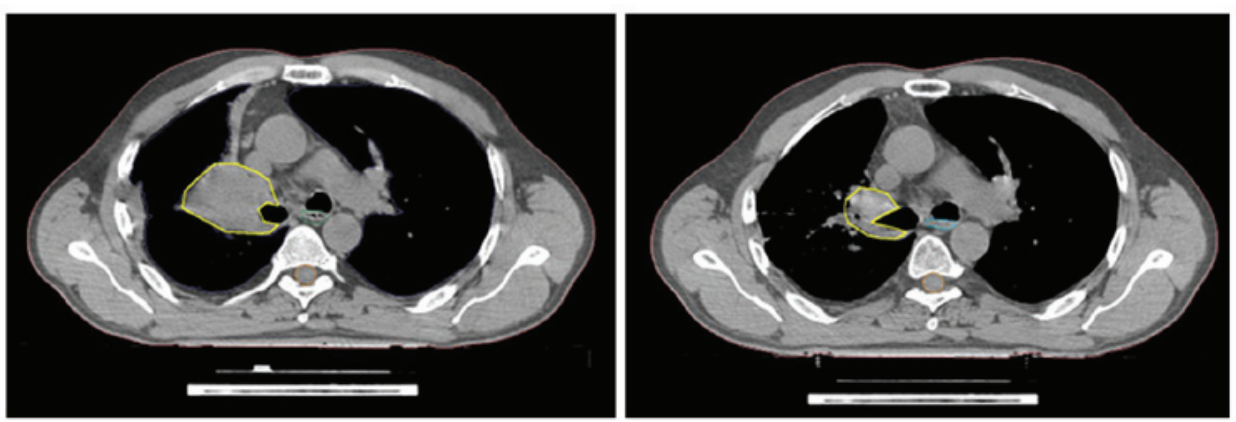

Figure 1. CT-based delineation of gross tumor volume (GTV). Representative thoracic CT scan (transversal plane) showing the cT4 tumor lesion (yellow circle) of a NSCLC patient in the initial planning CT before the start of radiotherapy (left) and in the boost planning CT after 40 Gy (right). CT, computed tomography.

Course of OPN and GTV and their correlation. Median pre-RT GTV1 was $90.4 \mathrm{ml}(3.3-270.9 \mathrm{ml})$ and after $40 \mathrm{~Gy}$, median GTV2 was $63 \mathrm{ml}$ which was significantly lower than GTV1 before $\mathrm{RT}(\mathrm{P}<0.0001)$. A positive correlation was noted between GTV1 (before RT) and GTV2 (at $40 \mathrm{~Gy}), \mathrm{r}=.7(\mathrm{P}<0.0001)$. The absolute GTV reduction was $38.9(-265-61.9) \mathrm{ml}$ during RT (GTV1 to GTV2) and highly significant $(\mathrm{P}<0.0001)$. The relative reduction of GTV during RT was $-42 \%(-103-99 \%)(\mathrm{P}<0.0001)$.

Median OPN before ( $\mathrm{t} 0)$, at the end of (t1) and four weeks after RT (t2) was 846 (361-2441), 777.4 (323-1397.7) and 623.5 (71.6-2248) $\mathrm{ng} / \mathrm{ml}$. Significant correlations could be determined between OPN t1 and $\mathrm{t} 2(\mathrm{r}=0.6 ; \mathrm{P}=0.005)$ and between relative OPN plasma level changes during RT (t0 to $\mathrm{t} 1$ ) and after RT (t1 to t2, r=0.8; $\mathrm{P}<0.0001$ ). Median OPN plasma levels before $(\mathrm{t} 0)$ and at the end of RT (t1) were not significantly different $(\mathrm{P}=0.46)$ but $\mathrm{OPN}$ plasma levels 4 weeks after RT ( $\mathrm{t} 2)$ were significantly lower than plasma levels at the end of RT (t1, $\mathrm{P}=0.005$ ). During RT (t0 to t1), absolute median OPN decline was $56.1(-1732.7-616.8) \mathrm{ng} / \mathrm{ml}$ and after RT it was 54.1 $(-761-1155.3) \mathrm{ng} / \mathrm{ml}$. The relative OPN plasma level reduction during RT (t0 to t1) was -7.9\% (-71-147.6\%) and after RT (t1 to t2) it was $-20.5 \%(-92.9-225.2 \%)$. However, these relative changes remained insignificant $(\mathrm{P}=0.37$ and 0.74$)$.

No correlation was observed neither between absolute pre-RT OPN and GTV1 values (Fig. 2A; P=.97) nor between absolute OPN plasma levels at the end of RT (OPN t1) and GTV2 $(\mathrm{P}=0.9$ ). Relative OPN plasma level changes during RT ( $\mathrm{t} 0$ to t1) also did not correlate with GTV changes during RT (GTV1 to GTV2), $P=0.14$. In addition, cross table analysis revealed no significant interrelation between OPN and GTV changes during radiotherapy ( $\mathrm{P}=0.1)$ (Fig. 2B; Table II).

Prognostic interrelation of OPN and GTV. Median follow-up in surviving patients was 65 (28-66) months. By the time of last survival data update, 24 patients $(89 \%)$ already had died. Median OS was 19.8 (2-66) months and 3-year survival rate was $17 \%$.

On univariate analysis, higher grade $(\mathrm{P}=0.009)$, T-stage $(\mathrm{P}=0.005), \mathrm{N}$-stage $(\mathrm{P}=0.03)$ and UICC-stage $(\mathrm{P}=0.02)$ were associated with inferior OS. GTV2 significantly predicted OS $(\mathrm{P}=0.03)$ which was 12.2 (9.8-14.6) months in patients with high GTV2 compared to 29.6 (18.3-40.9) months in patients with low GTV2. Pre-RT GTV1 trended to be related to OS $(\mathrm{P}=.08)$ with an elevated risk to die for patients with high baseline GTV1 $(\mathrm{rr}=2.1[0.9-5.1], \mathrm{P}=0.08)$.
Absolute OPN plasma levels at either time point ( $\mathrm{t} 0, \mathrm{t} 1$, t2; $\mathrm{P}=0.25,0.63,0.77)$, relative OPN plasma level changes during (t0 to t1, $\mathrm{P}=0.78$ ) and after $\mathrm{RT}$ (t1 to t2, $\mathrm{P}=0.68$ ) and GTV changes during RT $(\mathrm{P}=0.49)$ were not related to $\mathrm{OS}$ in univariate analysis.

For multivariate analysis, OPN and GTV were evaluated together with known prognostic factors and parameters which were significantly associated with OS in univariate analysis.

Initially, we evaluated baseline GTV1 and OPN (t0). After a stepwise backward multivariate analysis, OPN t0 $(\mathrm{P}=0.001)$ and GTV1 $(\mathrm{P}=0.02)$ were independent predictors of OS besides age $(\mathrm{rr}=1.3$ [1.1-8.1], $\mathrm{P}=0.008)$, gender $(\mathrm{rr}=4.4$ [1.9-35.2], $\mathrm{P}=0.001)$, weight loss $(\mathrm{rr}=1.8[1.3-17.5], \mathrm{P}=0.001)$, grade $(\mathrm{rr}=1.3$ [1.8-8.2], $\mathrm{P}=0.001)$, T-stage $(\mathrm{rr}=2.2$ [1.9-10.1], $\mathrm{P}<0.001)$ and $\mathrm{N}$-stage $(\mathrm{rr}=3$ [1.7-14.6], $\mathrm{P}=0.02)$. We then analyzed OPN at the end of RT (t1) and GTV2 (after $50 \mathrm{~Gy}$ ) in the same initial prognostic model and found both OPN t1 $(\mathrm{P}<0.001)$ and GTV2 $(\mathrm{P}=0.001)$ to be significant predictors of OS.

When relative OPN and GTV changes were evaluated in multivariate analysis, OPN plasma level changes after (t1 to t2) and GTV changes during RT (GTV 1 to GTV 2) remained independent predictors for OS with a significantly elevated risk of death for people OPN plasma level increases after (OPN t1t2: $r r=4.2$ [0.24-71.5], $\mathrm{P}=0.02)$ or GTV increases during RT (GTV 1 to 2: $\mathrm{rr}=6.7$ [1.7-22.9], $\mathrm{P}=0.02$ ) besides grade ( $\mathrm{rr}=1.4$ [0.5-8.3], $\mathrm{P}=0.01)$, histology ( $\mathrm{rr}=1.2$ [1.1-6.7], $\mathrm{P}=0.01)$ and $\mathrm{N}$-stage ( $\mathrm{rr}=3$ [0.6-14.6], $\mathrm{P}=0.06)$. Median OS was $8.7(0-42.8)$ months in patients with increasing OPN plasma levels after RT compared to 31.4 (1.3-45.6) months in patients with decreasing post-treatment OPN (t1 to t2). In patients whose GTV declined during RT (from the start of RT until completion of $50 \mathrm{~Gy}$ ), median OS was 21.8 (4.2-35.5) while it was 2.3 (0-31.3) months in patients with increasing GTV.

When absolute GTV values before RT (GTV1 $>/<$ median) were evaluated together with GTV changes during RT $(>/<$ median \% change from GTV1 to GTV2), the best OS (33.9 [20.1-47.7] months, $\mathrm{P}=0.02$ ) could be observed in the subgroup with small pre-RT GTV1 (<median) and large GTV decrease (from GTV1 to GTV2) while the poorest survival was noted in patients with high initial GTV1 before RT and a GTV reduction during RT (GTV1 to GTV2) below the median (6.9 [2.1-11.7] months) (Fig. 3A). The combination of absolute pre-RT GTV1 and GTV change during RT remained a significant predictor of OS in multivariate analysis $(\mathrm{P}=0.03)$ besides $\mathrm{N}$-stage $(\mathrm{P}=0.02)$ and grade $(\mathrm{P}=0.04)$. 
Table I. Demographic and clinical characteristics of NSCLC patients $(n=27)$.

\begin{tabular}{|c|c|}
\hline Clinical characteristics & No. patients (\%) \\
\hline \multicolumn{2}{|l|}{ Treatment } \\
\hline Radiotherapy & $7(26)$ \\
\hline Radiochemotherapy & $20(74)$ \\
\hline \multicolumn{2}{|l|}{ Gender } \\
\hline Male & $23(85)$ \\
\hline Female & $4(15)$ \\
\hline \multicolumn{2}{|l|}{ Age } \\
\hline Median (range) & $64(47-86)$ \\
\hline \multicolumn{2}{|l|}{ Histology } \\
\hline Adeno & $11(40)$ \\
\hline $\mathrm{SCC}^{1}$ & $15(56)$ \\
\hline Large-cell & $1(4)$ \\
\hline \multicolumn{2}{|l|}{ Weight loss ${ }^{\mathrm{a}}$} \\
\hline Yes & $7(26)$ \\
\hline No & $20(74)$ \\
\hline \multicolumn{2}{|l|}{ Anemia } \\
\hline Yes & $13(81)$ \\
\hline No & $3(19)$ \\
\hline \multicolumn{2}{|l|}{ Hemoglobin, g/dl } \\
\hline Median (range) & $12.7(8.4-14.7)$ \\
\hline \multicolumn{2}{|l|}{ FEV $1^{\mathrm{b}}$} \\
\hline Median (range) & $67.6(36.4-106.1)$ \\
\hline \multicolumn{2}{|l|}{ Tumor grade } \\
\hline Well (G1) & $2(7)$ \\
\hline Moderate (G2) & $5(19)$ \\
\hline Poor (G3) & $14(52)$ \\
\hline Undifferentiated (G4) & $1(3)$ \\
\hline Unknown & $5(19)$ \\
\hline \multicolumn{2}{|l|}{ T-stage } \\
\hline $\mathrm{T} 1$ & $1(4)$ \\
\hline $\mathrm{T} 2$ & $9(33)$ \\
\hline $\mathrm{T} 3$ & $5(19)$ \\
\hline $\mathrm{T} 4$ & $12(44)$ \\
\hline \multicolumn{2}{|l|}{ N-stage } \\
\hline No & $2(7)$ \\
\hline N1 & $0(0)$ \\
\hline $\mathrm{N} 2$ & $15(56)$ \\
\hline N3 & $10(37)$ \\
\hline \multicolumn{2}{|l|}{ UICC-stage } \\
\hline IIA & $1(4)$ \\
\hline IIB & $10(37)$ \\
\hline IIIA & $16(59)$ \\
\hline
\end{tabular}

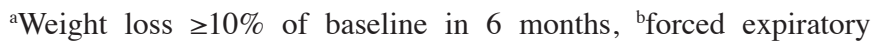
one-second volume in $\%$ of normal value. SCC, squamous cell carcinoma; UICC, union for international cancer control.

By combining baseline OPN (t0) plasma levels and GTV (1) before RT, a trend for superior OS (41.3 [9.3-73.3] months,
Table II. Cross table of the course (increase vs. decrease) of OPN and gross tumor volume (GTV) during radiotherapy.

\begin{tabular}{lrrr}
\hline & \multicolumn{2}{c}{ OPN t0 to t1 } & \\
\cline { 2 - 3 } & Decrease & Increase & Total \\
\hline GTV1 to GTV2 & & & \\
Decrease & 15 & 10 & 25 \\
Increase & 0 & 2 & 2 \\
Total & 15 & 12 & 27 \\
\hline
\end{tabular}

OPN, osteopontin.

$\mathrm{P}=0.08)$ was noted in the group with both low GTV1 and low OPN t0 (<median) before RT while patients with both high OPN t0 and GTV1 (>median) had the most inferior OS (12.2 [12.1-12.3]) (Fig. 3B). In multivariate analysis, together with other prognostic factors, the combination of OPN t0 plasma levels and GTV1 did not reach statistical significance.

The combination of relative changes in OPN plasma levels (t0 to t1) and GTV during RT (GTV1 to GTV2) was also related to prognosis. Patients with a decline in both OPN plasma levels (t0 to t1) levels and GTV during RT (GTV1 to GTV2) higher than the median had the best OS (29.6 [23.2-35.9] months) compared to patients with increases of both OPN (t0 to t1) and GTV (GTV1 to GTV2) higher than the median during RT who had the worst OS $(6.9$ [0-15.5], $\mathrm{P}=0.07)$. In the multivariate analysis with other known prognostic factors, combined OPN plasma level and GTV changes during RT remained insignificant.

\section{Discussion}

The prognostic relevance of elevated OPN plasma levels has been demonstrated in the chemotherapy $(18,19)$ and surgery of NSCLC (20), and also for tumor volume. Furthermore, there is strong evidence in the literature that GTV is a potential prognostic factor in conformal RT of NSCLC (13-16,21).

We previously demonstrated that both pre-treatment OPN and particularly OPN plasma level changes after RT are associated with prognosis in inoperable NSCLC treated by radical CCRT $(11,12)$. Interestingly, we also found a strong association of GTV with survival in the same patient collective. Assuming a possible prognostic quality of both OPN and GTV in the CCRT of NSCLC, it needs to be determined whether OPN plasma levels merely reflect tumor volume changes during RT, accounting for their prognostic effect. Thus, the present study aimed to investigate the interrelation of these two potential prognostic factors in definite CCRT of NSCLC.

In the current study, we identified a significant association of GTV2 (after 40 Gy) with survival outcome. GTV1 before RT trended to be associated with OS in univariate analysis which amends current literature showing strong evidence for a prognostic significance of tumor volume detected before RT (13-16,22) or chemotherapy of NSCLC (23). Koo et al (14) for instance reported a trend for inferior survival outcome in patients with poor tumor volume reduction during CCRT of 

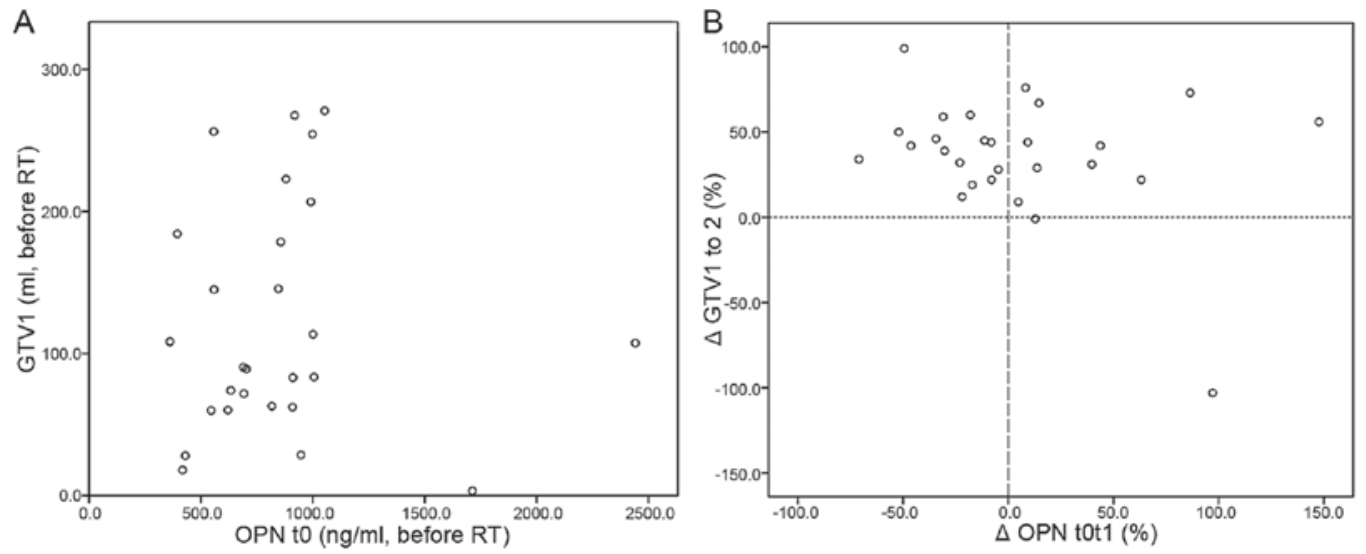

Figure 2. Interrelation of baseline OPN (t0) and GTV1 before radiotherapy (A), left. Dot plot of median baseline OPN plasma levels (t0, ng/ml) and gross tumor volume (GTV1, ml) before radiotherapy. (B) Relative changes of OPN plasma levels ( $\mathrm{t} 0$ to t1) and GTV during RT (GTV1 to GTV2, right. Dot plot of relative changes of OPN plasma levels (t0 to t1) and gross tumor volume (GTV1 to GTV2) during radiotherapy (\%). OPN, osteopontin; RT, radiotherapy.
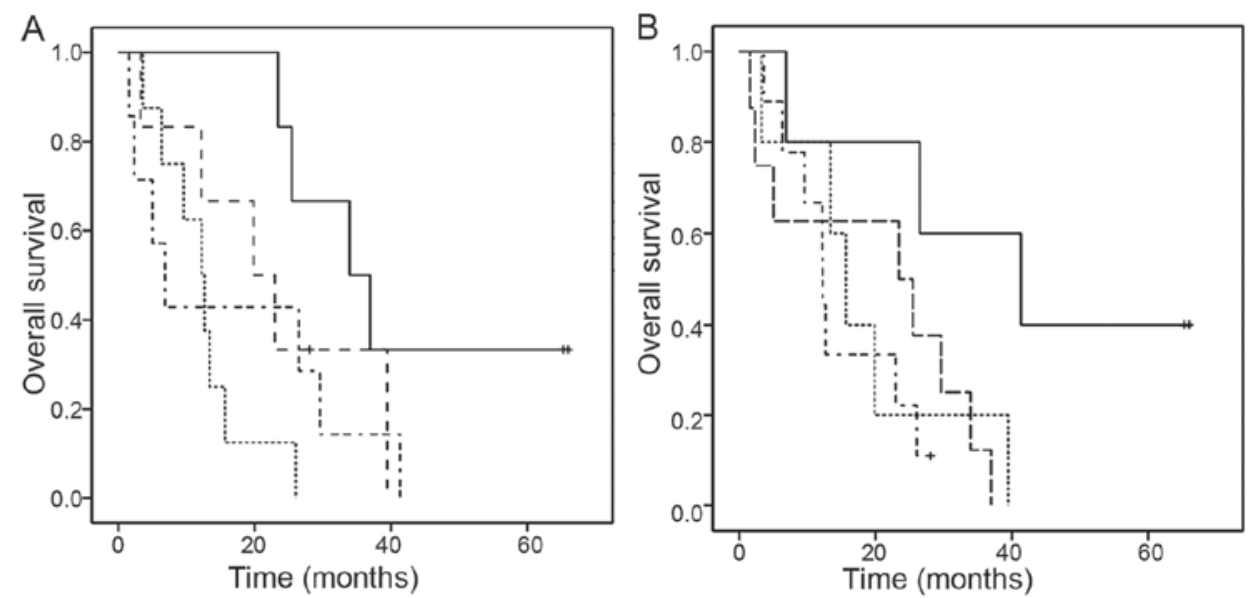

Figure 3. Prognostic significance of the combination of absolute GTV values before radiotherapy (GTV1) and relative GTV changes during radiotherapy (GTV1 to GTV2) and of the combination of baseline OPN (t0) and GTV1 before radiotherapy. (A) Kaplan-Meier plot of overall survival according to the combination of absolute GTV before radiotherapy (GTV1 $>/<$ median) and GTV change during radiotherapy (GTV1 to GTV2 $>/<$ median). Continuous line (GTV1 <median, GTV reduction during radiotherapy >median; n=6), long dashed line (GTV1 <median, GTV decrease during radiotherapy <median; n=6), dotted line (GTV1 >median, GTV reduction during radiotherapy $>$ median; n=8), dotted-dashed line (GTV $1>$ median, GTV reduction during radiotherapy $<$ median; n=7). (B) Kaplan-Meier plot of overall survival according to the combination of absolute GTV before radiotherapy (GTV1 $>/<$ median) and OPN before radiotherapy (OPN t0 $><<$ median). Continuous line (GTV1 and OPN t0 <median; n=5), long dashed line (GTV1 $>$ median, OPN t0 < median; n=8), dotted line (GTV1 <median, OPN t0 >median; n=5), dotted-dashed line (GTV1 and OPN t0 >median; n=9).

NSCLC, which is supported by our finding that GTV changes during RT were significantly associated with OS. In contrast to these and our own results, is the work of Ball et al (24) who did not find significant prognostic information provided by tumor volume in the RT of NSCLC.

In our exploratory multivariate analysis, relative OPN plasma level changes after and GTV changes during RT remained independent predictors of OS. When absolute OPN and GTV values were evaluated in multivariate an analysis, we found baseline OPN (t0) and GTV1 before RT as well as OPN at the end of RT (t1) and GTV2 (after 40 Gy) to be independent predictors of OS in different prognostic models. These results amend current literature such as the work of Yamane et al (25) who reported residual tumor volume after neoadjuvant chemotherapy of NSCLC to be prognostic, contrasting the results of Koo et al (14) who did not find a prognostic significance of post-RT GTV in the CCRT of NSCLC.
Interestingly, when we evaluated the combination of absolute GTV before RT and changes in GTV during RT, we found that patients with low initial GTV (before RT) and a significant decrease in GTV during RT had the best OS. Furthermore, the combination of baseline OPN t0 and GTV1 (before RT) was prognostically relevant, that is, patients with both high pre-RT OPN plasma levels and high GTV (>median), had the worst OS. In addition, patients with a pronounced decrease in both OPN plasma levels (t0 to t1) and GTV during RT (GTV1 to GTV2) had superior OS. Unlike the combination of absolute GTV before RT and GTV changes during RT however, which remained independent predictors of OS in multivariate analysis, the above mentioned combinations of pre-RT OPN t0 plasma levels and GTV1 or OPN plasma level and GTV changes during RT did not reach statistical significance. This suggests the hypothesis, that OPN plasma levels and GTV (both their changes and absolute values) are not interrelated in terms of prognosis, but do possess each parameter separately, a prognostic quality. 
However, the changes of OPN plasma levels during RT, that is from before RT (t0) to the end of RT (t1), do not depict the same time frame as GTV changes during radiotherapy, i.e. from before RT (GTV1) to 40 Gy (when GTV2 was re-contoured) which limits conclusions from a combined analysis of OPN plasma level and GTV changes during radiotherapy.

With definite CCRT being the standard approach in locally advanced, non-metastasized inoperable NSCLC, a GTV decline during RT could indicate a prognostically relevant early response to treatment $(14,26)$, while OPN plasma level increases after RT might be related to early tumor or metastasis regrowth (12).

The results of the present study were more robust when known clinical prognostic factors (27) were included in multivariate analysis together with OPN and GTV which is suggestive of heterogeneities of clinical prognostic factors and variable GTVs which may have masked to some extent survival differences in univariate analysis which also was restricted by the small overall patient number.

Bradley et al (13) noted a survival decrease with increasing GTV based on a size-dependent GTV classification. Due to the small patient number in our study, further classification of GTV into subgroups according to size would not have been reasonable. Thus, GTV changes in our study were split by the median as a cut-off value in our study which of course is a hypothetical cut-off value. Future studies could incorporate receiver operating characteristic (ROC) curves and statistical methods such as Contal and O'Quigleys (28) to test hypothetical cut-off value candidates and identify the most reliable cut-off value (14).

The present study did not specifically distinguish between tumor and nodal volume, since nodal volume in our patients was difficult to separate from tumor volume due to conglomerate with the primary. However, the prognostic role of a segregation of lymph nodes from primary tumors remains controversial $(14,15,21)$.

To the best of our knowledge, this is the first study to evaluate the correlation of OPN with GTV in CCRT of NSCLC. We found pre-RT OPN plasma levels not to be associated with pre-RT GTV. Despite the positive correlation of OPN plasma levels detected at different time points $(\mathrm{t} 0, \mathrm{t} 1, \mathrm{t} 2)$, no correlation between absolute OPN plasma levels and GTV values detected at different time points was found. A correlation between relative OPN plasma level changes and GTV changes could also not be determined which suggests that tumor volume (and its changes) does not affect OPN plasma levels.

We noted a significant reduction of GTV during RT and also OPN plasma levels declined both during and after RT. Together with the lack of a significant correlation between OPN and GTV, these results could indicate that these two parameters are independently affected by RT.

Initial GTV contouring in this study was PET-CT based and tumor volume therefore reflecting a viable tumor component. Considering the potential relation of OPN with tumor hypoxia in lung cancer $(11,12,29,30)$, the decrease in both OPN plasma levels and GTV which was noted during RT in this study could indicate a differential influence of radiation on OPN and GTV. A radiation-induced decrease in specifically hypoxic tumor volume might translate into a decline in OPN secretion and ultimately result in reduced overall OPN plasma levels. Assuming that only vital tumor cells are capable of producing and secreting OPN and given that necrotic or largely hypoxic tumor areas have a considerably decreased glucose uptake, decreasing GTV might merely reflect shrinkage of active metabolic tumor volume (MTV). As such, GTV and its changes might be related to tumor kinetics rather than to tumor oxygenation $(31,32)$. Also, post-RT PET contains not only viable tumor volume but also radiation induced tissue injury which has not been shown to influence OPN plasma levels.

A major restriction of our study however is, that unlike for initial GTV contouring which was CT-based but included diagnostic FDG-PET information, for GTV re-contouring after $40 \mathrm{~Gy}$, we used CT alone. Despite adjusting for confounding hyperdensities such as atelectasis, pneumonia and pleural effusion by an experienced radiation oncologist, differentiation between treatment-induced changes and persistent or recurrent tumor remained difficult (33). In addition, FDG-PET was not part of routine surveillance and tumor response evaluation after RT in our patient collective. Consequently, assessment of tumor volume by PET-CT imaging at the time points of OPN readings and during follow-up could enhance the value of univariate and multivariate analyses by increasing congruency of OPN and tumor volume detection. Additionally, functional PET imaging such as hypoxia-specific FMISO-PET is a reasonable complement for future studies (34) in order to delineate the differential effects of radiation on hypoxic and metabolic tumor volume.

It appears that OPN velocity also provides additional clinical information (35) so that in future studies, OPN plasma levels could be further classified by their velocity $(31,35)$.

A clear limitation of this study which was a hypothesis-generating one is its small size, reflecting stringent patient selection criteria which included a minimum follow-up of two years. Of note however is, the prospective nature of the study and the homogeneity of the patient cohort where only NSCLC patients in M0-stage with absence of distant metastasis (and available OPN plasma samples at all three time points and PET-CT based contoured GTV) were included. This is representative of a patient population considered most appropriate for curative-intent treatment where prognostic and predictive factors are valuable tools. Nevertheless, the small patient number in subgroups of univariate and multivariate analyses underlines their exploratory character and needs to be taken into consideration in the interpretation of the preliminary results presented here.

In conclusion, the lack of a significant association and correlation between OPN and GTV together with our finding that OPN and GTV remained independent predictors of survival outcome in multivariate analysis supports the hypothesis that OPN plasma levels may not be surrogate and thus (prognostically) independent of tumor volume and its changes during RT. This hypothesis is further strengthened by our finding that the combination of absolute GTV before RT and GTV changes during RT were related to prognosis both in univariate and multivariate analysis but combinations of OPN plasma levels and GTV (both absolute values and their changes) did not reach statistical significance.

Consequently, tumor volume (GTV) and OPN plasma levels (both their changes and absolute values) are not interrelated in 
terms of prognosis but do possess-each parameter separately-a prognostic quality in the radical CCRT of NSCLC which justifies further prospective studies incorporating a larger patient number in order to determine the prognostic information OPN plasma levels provide beyond tumor volume and know prognostic factors such as $\mathrm{T}$ - and $\mathrm{N}$-stage.

\section{Acknowledgements}

DV and CO conceived the study. CO and FS carried out patient selection, recruitment and clinical data acquisition. $\mathrm{CO}$ and TR performed blood sample collection. $\mathrm{CO}$ and $\mathrm{MB}$ processed blood samples and carried out OPN measurements. FS and DV were responsible for GTV delineation. CO performed the statistical data analyses and drafted the manuscript. DV and MB provided scientific advice. All authors proofread the manuscript.

\section{References}

1. Ferlay J, Soerjomataram I, Dikshit R, Eser S, Mathers C, Rebelo M, Parkin DM, Forman D and Bray F: Cancer incidence and mortality worldwide: Sources, methods and major patterns in GLOBOCAN 2012. Int J Cancer 136: E359-E386, 2015.

2. Aupérin A, Le Péchoux C, Rolland E, Curran WJ, Furuse K, Fournel P, Belderbos J, Clamon G, Ulutin HC, Paulus R, et al: Meta-analysis of concomitant versus sequential radiochemotherapy in locally advanced non-small-cell lung cancer. J Clin Oncol 28: 2181-2190, 2010.

3. Vaupel P and Mayer A: Hypoxia in cancer: Significance and impact on clinical outcome. Cancer Metastasis Rev 26: 225-239, 2007.

4. Nordsmark M, Bentzen SM, Rudat V, Brizel D, Lartigau E, Stadler P, Becker A, Adam M, Molls M, Dunst J, et al: Prognostic value of tumor oxygenation in 397 head and neck tumors after primary radiation therapy. An international multi-center study. Radiother Oncol 77: 18-24, 2005.

5. Le QT: Identifying and targeting hypoxia in head and neck cancer: A brief overview of current approaches. Int J Radiat Oncol Biol Phys 69 (Suppl 2): S56-S58, 2007.

6. Höckel M and Vaupel P: Biological consequences of tumor hypoxia. Semin Oncol 28 (2 Suppl 8): S36-S41, 2001

7. Zips D, Böke S, Kroeber T, Meinzer A, Brüchner K, Thames HD, Baumann $M$ and Yaromina A: Prognostic value of radiobiological hypoxia during fractionated irradiation for local tumor control. Strahlenther Onkol 187: 306-310, 2011.

8. Bache M, Kappler M, Said HM, Staab A and Vordermark D: Detection and specific targeting of hypoxic regions within solid tumors: Current preclinical and clinical strategies. Curr Med Chem 15: 322-338, 2008

9. Le QT and Courter D: Clinical biomarkers for hypoxia targeting. Cancer Metastasis Rev 27: 351-362, 2008.

10. Vordermark D and Brown JM: Endogenous markers of tumor hypoxia predictors of clinical radiation resistance? Strahlenther Onkol 179: 801-811, 2003.

11. Ostheimer C, Bache M, Güttler A, Kotzsch M and Vordermark D: A pilot study on potential plasma hypoxia markers in the radiotherapy of non-small cell lung cancer. Osteopontin, carbonic anhydrase IX and vascular endothelial growth factor. Strahlenther Onkol 190: 276-282, 2014.

12. Ostheimer C, Bache M, Güttler A, Reese T and Vordermark D: Prognostic information of serial plasma osteopontin measurement in radiotherapy of non-small-cell lung cancer. BMC Cancer 14 $858,2014$.

13. Bradley JD, Leumwananonthachai N, Purdy JA, Wasserman TH, Lockett MA, Graham MV and Perez CA: Gross tumor volume, critical prognostic factor in patients treated with three-dimensional conformal radiation therapy for non-small-cell lung carcinoma. Int J Radiat Oncol Biol Phys 52: 49-57, 2002.

14. Koo TR, Moon SH, Lim YJ, Kim JY, Kim Y, Kim TH, Cho KH, Han JY, Lee YJ, Yun T, et al: The effect of tumor volume and its change on survival in stage III non-small cell lung cancer treated with definitive concurrent chemoradiotherapy. Radiat Oncol 9: $283,2014$.
15. Werner-Wasik M, Swann RS, Bradley J, Graham M, Emami B, Purdy J and Sause W: Increasing tumor volume is predictive of poor overall and progression-free survival: Secondary analysis of the Radiation Therapy Oncology Group 93-11 phase I-II radiation dose-escalation study in patients with inoperable non-small-cell lung cancer. Int J Radiat Oncol Biol Phys 70: 385-390, 2008.

16. Basaki K, Abe Y, Aoki M, Kondo H, Hatayama Y and Nakaji S: Prognostic factors for survival in stage III non-small-cell lung cancer treated with definitive radiation therapy: Impact of tumor volume. Int J Radiat Oncol Biol Phys 64: 449-454, 2006.

17. Cox DR: Regression models and life tables. J Royal Stat Soc Series B (Methodological) 34: 187-220, 1972.

18. Isa S, Kawaguchi T, Teramukai S, Minato K, Ohsaki Y, Shibata K, Yonei T, Hayashibara K, Fukushima M, Kawahara M, et al: Serum osteopontin levels are highly prognostic for survival in advanced non-small cell lung cancer: Results from JMTO LC 0004. J Thorac Oncol 4: 1104-1110, 2009.

19. Mack PC, Redman MW, Chansky K, Williamson SK, Farneth NC, Lara PN Jr, Franklin WA, Le QT, Crowley JJ, et al: Lower osteopontin plasma levels are associated with superior outcomes in advanced non-small-cell lung cancer patients receiving platinum-based chemotherapy: SWOG Study S0003. J Clin Oncol 26: 4771-4776, 2008.

20. Blasberg JD, Pass HI, Goparaju CM, Flores R, Lee S and Donington JS: Reduction of elevated plasma osteopontin levels with resection of non-small-cell lung cancer. J Clin Oncol 28: 936-941, 2010.

21. Alexander BM, Othus M, Caglar HB and Allen AM: Tumor volume is a prognostic factor in non-small-cell lung cancer treated with chemoradiotherapy. Int J Radiat Oncol Biol Phys 79: 1381-1387, 2011.

22. Kozak MM, Murphy JD, Schipper ML, Donington JS, Zhou L, Whyte RI, Shrager JB, Hoang CD, Bazan J, Maxim PG, et al: Tumor volume as a potential imaging-based risk-stratification factor in trimodality therapy for locally advanced non-small cell lung cancer. J Thorac Oncol 6: 920-926, 2011.

23. Stinchcombe TE, Morris DE, Moore DT, Bechtel JH, Halle JS, Mears A, Deschesne K, Rosenman JG and Socinski MA: Post-chemotherapy gross tumor volume is predictive of survival in patients with stage III non-small cell lung cancer treated with combined modality therapy. Lung Cancer 52: 67-74, 2006.

24. Ball DL, Fisher RJ, Burmeister BH, Poulsen MG, Graham PH, Penniment MG, Vinod SK, Krawitz HE, Joseph DJ, Wheeler GC and McClure BE: The complex relationship between lung tumor volume and survival in patients with non-small cell lung cancer treated by definitive radiotherapy: A prospective, observational prognostic factor study of the Trans-Tasman radiation oncology group (TROG 99.05). Radiother Oncol 106: 305-311, 2013.

25. Yamane Y, Ishii G, Goto K, Kojima M, Nakao M, Shimada Y, Nishiwaki Y, Nagai K, Kohrogi H and Ochiai A: A novel histopathological evaluation method predicting the outcome of non-small cell lung cancer treated by neoadjuvant therapy: The prognostic importance of the area of residual tumor. J Thorac Oncol 5: 49-55, 2010.

26. Soliman M, Yaromina A, Appold S, Zips D, Reiffenstuhl C, Schreiber A, Thames HD, Krause M and Baumann M: GTV differentially impacts locoregional control of non-small cell lung cancer (NSCLC) after different fractionation schedules: Subgroup analysis of the prospective randomized CHARTWEL trial. Radiother Oncol 106: 299-304, 2013.

27. Wigren T: Confirmation of a prognostic index for patients with inoperable non-small cell lung cancer. Radiother Oncol 44: 9-15, 1997.

28. Contal C and O'Quigley J: An application of changepoint methods in studying the effect of age on survival in breast cancer. Comput Stat Data Anal 30: 253-270, 1999.

29. Le QT, Kong C, Lavori PW, O'byrne K, Erler JT, Huang X, Chen Y, Cao H, Tibshirani R, Denko N, et al: Expression and prognostic significance of a panel of tissue hypoxia markers in head-and-neck squamous cell carcinomas. Int J Radiat Oncol Biol Phys 69: 167-175, 2007.

30. Le QT, Chen E, Salim A, Cao H, Kong CS, Whyte R, Donington J, Cannon W, Wakelee H, Tibshirani R, et al: An evaluation of tumor oxygenation and gene expression in patients with early stage non-small cell lung cancers. Clin Cancer Res 12: 1507-1514, 2006

31. Chu KP, Murphy JD, La TH, Krakow TE, Iagaru A, Graves EE, Hsu A, Maxim PG, Loo B, Chang DT and Le QT: Prognostic value of metabolic tumor volume and velocity in predicting head-and-neck cancer outcomes. Int J Radiat Oncol Biol Phys 83: 1521-1527, 2012. 
32. Lee P, Bazan JG, Lavori PW, Weerasuriya DK, Quon A, Le QT, Wakelee HA, Graves EE and Loo BW: Metabolic tumor volume is an independent prognostic factor in patients treated definitively for non-small-cell lung cancer. Clin Lung Cancer 13: $52-58,2012$

33. Choi YW, Munden RF, Erasmus JJ, Park KJ, Chung WK, Jeon SC and Park CK: Effects of radiation therapy on the lung: Radiologic appearances and differential diagnosis. Radiographics 24: 985-998, 2004.
34. Zips D, Zöphel K, Abolmaali N, Perrin R, Abramyuk A, Haase R, Appold S, Steinbach J, Kotzerke J and Baumann M: Exploratory prospective trial of hypoxia-specific PET-imaging during radiochemotherapy in patients with locally advanced head-and-neck cancer. Radiother Oncol 105: 21-28, 2012.

35. Joseph S, Harrington R, Walter D, Goldberg JD, Li X, Beck A, Litton T, Hirsch N, Blasberg J, Slomiany M, et al: Plasma osteopontin velocity differentiates lung cancers from controls in a CT screening population. Cancer Biomark 12: 177-184, 2012. 